\title{
RIGHT HANDED WEAK CURRENTS IN SUM RULES FOR AXIALVECTOR CONSTANT RENORMALIZATION
}

\author{
N.B.Shul'gina \\ The Niels Bohr Institute, \\ Blegdamsvej 17, DK-2100 Copenhagen Ø,Denmark*
}

\begin{abstract}
The recent experimental results on deep inelastic polarized lepton scattering off proton, deuteron and ${ }^{3} \mathrm{He}$ together with polarized neutron $\beta$-decay data are analyzed. It is shown that the problem of Ellis-Jaffe and Bjorken sum rules deficiency and the neutron paradox could be solved simultaneously by assuming the small right handed current (RHC) admixture in the weak interaction Lagrangian. The possible RHC impact on pion-nucleon $\sigma$-term and Gamow-Teller sum rule for $(p, n)$ nuclear reactions is pointed out.
\end{abstract}

PACS numbers: $12.60 \mathrm{Cn}, 11.55 \mathrm{Hx}, 12.15 \mathrm{Ji}, 24.80 \mathrm{Ba}$

In comparing sum rules for axialvector constant renormalization with the experimental data one usually assumes that the axialvector constant renormalization $\lambda=g_{A} / g_{V}$ is a well known value, measured with high accuracy in neutron beta decay. Is it really the case? Let us consider the modern experimental status of neutron beta decay in more details. The axialvector constant renormalization can be extracted either from the neutron life time $\left(t_{n}\right)$ or from the electron asymmetry $(A)$ according to the well known formulae:

$$
f_{n} t_{n}=2(f t)_{0-0} /\left(1+3 \lambda_{\tau}^{2}\right)
$$

*Permanent address: The Kurchatov Institute, 123182 Moscow, Russia 


$$
A=-2 \lambda_{c}\left(\lambda_{c}+1\right) /\left(1+3 \lambda_{c}^{2}\right)
$$

In the Standard Model of the electroweak interaction $\lambda_{\tau}$ should be equal to $\lambda_{c}$. According to the recent experimental data on the polarized neutron beta decay, which was measured with an accuracy about $10^{-3}$, and the experimental data on $0^{+}-0^{+}$beta transitions $[1,2], \lambda_{\tau}$ and $\lambda_{c}$ differ from each other at $2.6 \sigma$ level. Indeed, for the electron asymmetry $A=-0.1126 \pm 0.0011[3], \tau_{n}=$ $886.7 \pm 1.5 \mathrm{~s}$ (mean-weighted value of Particle Data Group data [3] and recently appeared value of ref. [4]) and $f t_{0-0}=3074.0 \pm 3.5 \mathrm{~s}$ [1]:

$$
\begin{aligned}
& \lambda_{\tau}=-1.270 \pm 0.002 \\
& \lambda_{c}=-1.257 \pm 0.003
\end{aligned}
$$

In the papers $[5,6]$ the neutron paradox was explained in the framework of the left-right symmetric model $S U(2)_{L} \times S U(2)_{R} \times U(1)$ [7] and the possibility of the RHC admixture in the neutron beta decay was pointed out. It should be stressed that the vector and the axial-vector constants are "renormalized" by RHC in a different way, so that the "nucleonic" or "bare" axialvector constant renormalization $\lambda_{N}$ may differ significantly from well known value $\lambda=-1.26[5,6]$.

In the the simplest manifestly left-right symmetric model of $S U(2)_{L} \times$ $S U(2)_{R} \times U(1)$ the expressions for $\lambda_{\tau}$ and beta decay asymmetry $A$ have the following form [5]:

$$
\begin{array}{r}
\lambda_{\tau}=\lambda_{N}[(Z+X) /(Z-X)]^{1 / 2} \\
A=-2 \lambda_{N}\left(\lambda_{N}+1+Y \lambda_{N}\right)\left(1-X^{2}\right)^{1 / 2} /\left[(Z-X)\left(1+3 \lambda_{\tau}^{2}\right)\right]
\end{array}
$$

where

$$
\begin{gathered}
X=\sin 2 \zeta, Y=(1-\eta) \sin 2 \zeta /(1+\eta), \\
Z=\left(1+\eta^{2}\right) /\left(1-\eta^{2}\right)
\end{gathered}
$$

Both experimental values are functions of the model parameters, $\eta, \zeta, \lambda_{N}$, which have the following physical meaning: $\eta=\left(M_{1} / M_{2}\right)^{2}$ denotes the squared mass ratio of the $W_{1}$ and $W_{2}$ bosons; $\zeta$ - the mixing angle of these bosons $\left(W_{L}=W_{1} \cos \zeta+W_{2} \sin \zeta, W_{R}=-W_{1} \sin \zeta+W_{2} \cos \zeta\right) ; \lambda_{N}$ - the bare relative renormalization of the axialvector nucleon current. It is important to stress here that the $\lambda_{N}$ in Eq.(5-6) is the value, which should be 
determined in a way independent of the nature of weak interaction e.g.,from Adler-Weisberger sum rule.

In the $S U(2)_{L} \times U(1)$ limit the parameters tend to :

$$
\eta=0, \zeta=0, \lambda_{N}=\lambda_{\tau}=\lambda_{c}
$$

The set of the experimental data on the $\lambda_{\tau}, A, \mu$-decay [8] enables to restrict the range of permissible values of the $\lambda_{N}, \zeta, \eta$ parameters.

$$
\begin{gathered}
0.003 \leq \zeta \leq 0.054, \eta \leq 0.036,-1.265 \leq \lambda_{N} \leq-1.131 \\
-0.054 \leq \zeta \leq-0.020, \eta \leq 0.024,-1.415 \leq \lambda_{N} \leq-1.20 \\
M_{W R} \geq 427 G e V(98 \% \text { c.l. })
\end{gathered}
$$

As is seen from Eq.(9), in each of two regions, $\lambda_{N}$ varies within $10 \%$ interval. The muon data [8] does not indicate the RHC and is used here in order to restrict upper limits of the $\zeta$ and $\eta$ parameters, while the neutron data $[3,4]$ provides the lower limits. In principle, the muon data can be used only if the muon-electron universality is assumed.

The nonzero contribution of RHC in nuclear beta decay was confirmed also by the joint analysis of neutron and ${ }^{19} \mathrm{Ne}$ beta decay [9]. It is necessary to notice that in the simplest left- right symmetric model the large mixing angles $(\zeta \geq 0.006)$ disagree with the unitarity of the Cabbibo-KobayashiMaskawa matrix for three quark generations. However this can be avoided in an extended version of the model. Anyway, the unitarity problem is a theoretical one, while in this paper only experimental data will be analyzed.

\section{Ellis-Jaffe sum rule}

An interesting aspect of the RHC arises when considering the EMC experimental data on deep inelastic polarized lepton- proton scattering. Let us recall the essence of the problem. EMC measurements of the spin dependent proton structure function [10]:

$$
\Gamma^{p}=\int_{0}^{1} g^{p}(x) d x=0.126 \pm 0.010 \pm 0.015
$$

indicated a significant deviation from Ellis-Jaffe sum rule [11], which was derived using $S U(3)$ current algebra with the assumption of unpolarized strange quark sea:

$$
\Gamma^{p}=\int_{0}^{1} g^{p}(x) d x=\frac{1}{12}\left|\frac{g_{A}}{g_{V}}\right|\left(1+\frac{5}{3} \frac{3 F-D}{F+D}\right)
$$


where $F$ and $D$ are the $S U(3)$ invariant matrix elements of the axial current, which are usually deduced as a weighted mean values of all types of hyperon semileptonic beta decay data.

After correcting for the QCD radiative effects [12] the integral (11) becomes [10]:

$$
\Gamma^{p}=0.189 \pm 0.005,
$$

where the old value $g_{A} / g_{V}=1.254 \pm 0.006$ from neutron beta decay and $F / D=0.632 \pm 0.024$ from overall hyperon beta decay fit were used. The discrepancy (more than two standard deviations) between predicted (12) and experimental (10) values, known as the "proton spin crisis", created a lot of theoretical explanations ( see, for example, the review [13] and references therein). In the most of them the proton spin is supposed to be carried by gluons or orbital angular motion. However these models can't, probably, explain polarized proton - nucleon data at high energies (for details see ref.[14]).

The RHC impact was not considered as a possible explanation although this seems to be simplest. Indeed, $g_{A} / g_{V}$ in the Ellis- Jaffe sum rule is just the same $\lambda_{N}$ value considered above. Measured values, say $\lambda_{\tau}$, can be affected by RHC as seen from Eq.(5). As is mentioned above, $\lambda_{N}$, in principle, should not be equal to $\lambda_{\tau}$ or $\lambda_{c}$, extracted from the neutron life time or the electron asymmetry, especially if one takes into account the $2.6 \sigma$ discrepancy between $\lambda_{\tau}$ and $\lambda_{c}$. If one supposes that the RHC do exist in the neutron beta decay, the sum rules in deep inelastic lepton- nucleon scattering should be tested in another way. First of all, one should realize that the $g_{A} / g_{V}$ value in Eq. (11) is neither $\lambda_{\tau}$ nor $\lambda_{c}$ nor their mean value, but should be taken from Eq. (5). Secondly, the $F / D$ value can differ from the least square fit to all hyperon decay data, which can be affected by RHC in different ways. Strictly speaking, for a precise estimation of the $F / D$ value all hyperon decay data should be revised in the framework of $S U(2)_{L} \times S U(2)_{R} \times U(1)$ model. A thorough analysis of hyperon $\beta$ decays for the case of RHC is tedious and will be done elsewhere. Perhaps the shortest and rather accurate approach to the problem is to rewrite Eq.(11) in the following form:

$$
\Gamma^{p}=\left\{\frac{2}{9}\left|\frac{g_{A}}{g_{V}}\right|^{n p}-\frac{5}{18}\left|\frac{g_{A}}{g_{V}}\right|^{\Sigma n}\right\}[(Z-X) /(Z+X)]^{1 / 2}
$$

where indices $n p$ and $\Sigma n$ mean that the ratio relates to the neutron $\beta$-decay 
and to $\Sigma^{-} \rightarrow n+e^{-}+\bar{\nu}$ decay, respectively. Eq.(13) can be easily derived using the $\mathrm{SU}(3)$ matrix elements for neutron and $\Sigma^{-}$- hyperon $\beta$ - decay: $\left(g_{A} / g_{V}\right)^{n p}=F+D ;\left(g_{A} / g_{V}\right)^{\Sigma n}=D-F$.

Two high statistic experiments $[15,16]$ with unpolarized beams give | $g_{A} /\left.g_{V}\right|^{\Sigma n}=0.36 \pm 0.04$. Using this value, $\left|g_{A} / g_{V}\right|^{n p}=\left|\lambda_{\tau}\right|=1.270 \pm$ 0.002 and correcting for QCD radiative effects one can obtain:

$$
\Gamma^{p}=(0.170 \pm 0.010)[(Z-X) /(Z+X)]^{1 / 2}
$$

When the RHC parameter $\zeta$ varies within the interval $0.022 \leq \zeta \leq 0.054$, which is permitted by neutron and muon beta decay data, the new version of Ellis- Jaffe sum rule (13) agrees with the experimental data.

\section{Bjorken sum rule}

A more fundamental sum rule for deep inelastic polarized lepton- nucleon scattering is the Bjorken sum rule, since $S U(3)$ symmetry is not assumed. This sum rule relates the integral over $x$ of the difference of neutron and proton structure functions to the bare axialvector constant renormalization in the following way:

$$
\int_{0}^{1}\left(g^{p}(x)-g^{n}(x)\right) d x=\frac{1}{6}\left|\frac{g_{A}}{g_{V}}\right|
$$

So an observation of the spin dependent structure function of a neutron could be the less ambiguous way to estimate the bare axialvector constant renormalization. The neutron spin structure function was determined very recently by measuring the asymmetry in deep inelastic scattering of polarized electrons from a polarized ${ }^{3} \mathrm{He}$ target [17]. Experimental $\Gamma^{n}$ value is found to be $-0.22 \pm 0.11$. Together with proton structure function (11), corrected for relevant $Q^{2}$, and Bjorken sum rule (14) it gives :

$$
0.833 \leq\left|\lambda_{N}\right| \leq 1.187(68 \% \text { c.l. })
$$

(with the mean value being equal to unity!). In terms of RHC parameters it means:

$$
0.03 \leq \zeta \leq 0.17
$$

which does not contradict to neutron and muon data. 
Measurements, carried out with an accuracy better than $10 \%$, could throw a light on the nature of Ellis- Jaffe and Bjorken sum rule violation. In any case, even for more precise future experiments a reasonable difference ( $\approx 10 \%$ ) between axialvector constant renormalization, extracted from the Bjorken sum rule, and that from the neutron lifetime can take place and can be explained in terms of RHC.

\section{Pion-nucleon $\sigma$ - term}

The experimental value of the pion decay constant $F_{\pi}$, deduced from the weak pion decay: $\pi \rightarrow \mu+\nu_{\mu}$ could be also renormalized by RHC. Indeed in the Standard model the pion decay is helicity suppressed and takes place only due to the muon mass. If the admixture of RHC is allowed, the decay probability increases. This means that the experimental value $F_{\pi \exp }$ is greater than bare one, $F_{\pi B}$.

After this remark, let us reanalyze the pion-nucleon $\sigma$ term. Along with EMC experimental data on deep inelastic polarized muon- proton scattering, a large experimental value of the pion- nucleon $\sigma$-term is considered as an evidence for large strange quark content of proton [18]. In the light of $F_{\pi}$ renormalization by RHC the surprisingly large experimental value of the pion-nucleon $\sigma$ term, as compared with the theoretical one under assumption of zero strange quark sea component of proton, can be explained. Keeping aside the details of calculations, as well an experimental uncertainties, analyzed thoroughly in Ref.[19], let us consider the treatment of experimental data. Actually ( for details see the review of E.Reya [20]) one can extract from experimental data only the ratio: $\sigma_{\pi N} / F_{\pi B}^{2}$. If one assumes that $F_{\pi}$ is renormalized by RHC in the same way as $\lambda_{N}$ (that is true for the simplest version of the left-right symmetric model), then

$$
F_{\pi B} \approx F_{\pi \exp }(1-2 \zeta)
$$

and the experimental values $\sigma_{\pi N}=45 \pm 10 \mathrm{MeV}$ [21] can be reconciled with theoretical values $\sigma_{\pi N}=23 \pm 5[19]$ within $1 \sigma$ interval for $\zeta \approx 0.05$., a value which explains also the Ellis-Jaffe sum rule deficiency, as well as the neutron paradox.

\section{Gamow-Teller sum rule for nuclear reactions}


There is a well known experimental fact [ 22 ], that in $(p, n)$ reactions on nuclei the Gamow-Teller sum rule [23]

$$
S^{+}(G T)-S^{-}(G T)=3 \lambda^{2}(N-Z)
$$

is not exhausted at low excitation energies including the Gamow-Teller giant resonance when one uses $\lambda=\lambda_{\tau}$. The deficiency or, so called "quenching", is about $40 \%$. The sum rule (15) is model independent if non nucleonic degrees of freedom are not introduced and isospin is a perfect symmetry. Different aspects of the nuclear structure as well as delta-isobar excitations were intensively discussed as a possible explanation of the quenching effect [24]. But if one takes into account that in the strong processes $\lambda$ in Eq.(15) is the bare value and should not be taken from neutron life time one half of the missing strength can be explained by the $\zeta \approx 0.05$, a value which explains the Ellis Jaffe and Bjorken sum rules deficiency as well as the pion-nucleon $\sigma$ - term and the neutron paradox. If RHC do exist one can obtain the relation:

$$
\left[S^{+}(G T)-S^{-}(G T)\right]_{\text {strong }} /\left[S^{+}(G T)-S^{-}(G T)\right]_{\text {weak }} \approx(1-4 \zeta)
$$

which is independent of nuclear structure models and enables, in principle, to deduce the RHC parameter $\zeta$ by comparing the "strong" and "weak" experimental GT strengths in the same region of excitation energies. Of course, the experimental uncertainties have to be at least less than $20 \%$. It should be noticed here, that in contrast to Eq.(16), the procedure of the GT strength extraction from the $(\mathrm{p}, \mathrm{n})$ reaction cross sections involves nuclear structure parameters. Therefore it would be worthy to test the prediction (16) in lightest nuclei, where the nuclear structure is calculated more reliably.

The pure $\lambda_{N}$ can be deduced from the experimental data, if one uses the Adler- Weisberger sum rule $[25,26]$ :

$$
1-\frac{1}{\lambda_{N}^{2}}=\frac{4 M}{g_{\pi n n}^{2}} \frac{1}{\pi} \int_{M_{N}+m_{\pi}}^{\infty} \frac{W d W}{W^{2}-M_{N}^{2}}\left[\sigma_{0}^{+}(W)-\sigma_{0}^{-}(W)\right]
$$

where $\sigma_{0}^{ \pm}$is the total cross section for scattering of a zero mass $\pi^{ \pm}$on a proton at the center of mass energy W.

Up to now, only two estimations of the $\lambda_{N}$ value from this relation exist: one, given by Adler [25] $-\lambda_{N}=-1.24$ and another, given by Weisberger $[26]-\lambda_{N}=-1.15$. Both estimations were done in 1965. Since that time new experimental data on pion -proton scattering has appeared and strong 
interaction constant $g_{\pi n n}$, has been revised. Therefore it would be worthwhile to reanalyze Adler- Weisberger sum rule in order to extract bare axialvector constant renormalization more accurately.

The problem of the bare value of the axialvector constant renormalization is important also for calculations of the counting rates in solar neutrino de-

tectors, which employ $(\mathrm{p}, \mathrm{n})$ experimental data for weak process calculations (see, e.g. [27]).

In conclusion, it should be emphasized, that intimate connection between low energy weak processes and high energy scattering processes, based on current algebra, provides sensitive tests for the Standard model of strong and electroweak interactions.

I am grateful to Yu.Gaponov, P.Herczeg, W.Weise, C.Gaarde, I.Towner and J.Bang for useful discussions. I am also thankful for the hospitality of The Niels Bohr Institute and NORDITA where part of this work has been done.

\section{References}

[1] I.S.Towner, Nucl.Phys. 540A(1992)478; F.C.Barker et.al, Nucl. Phys. 540A (1992) 501

[2] J.C.Hardy et.al Nucl.Phys.509A (1990) 429

[3] Particle Data Group Phys.Rev.D45 S1 (1992)

[4] W.Mampe, L.Bondarenko,V.Morozov, Yu.Panin, A.Fomin, JETP Lett. 57, N 2 (1993) 82

[5] Yu.V. Gaponov, N.B.Shul'gina, Yad.Fiz. 49 (5) (1989) 1359; "Weak and Electromagnetic Interactions in Nuclei" Proc. Int. Symp., Montreal, (1989) p.577

[6] Yu.V. Gaponov, N.B.Shul'gina, P.E.Spivak, Preprint IAE -5032/2 (1990); Yad.Fiz. 52 (6(12)) (1990) 1653; Phys. Lett. B253 (1991) 283

[7] Pati J.C. and A.Salam, Phys. Rev. D10 (1974) 275; R.N.Mohapatra, J.C.Pati, Phys. Rev. D11 (1975) 566; 2558 
[8] A.Jodidio et.al., Phys. Rev. D37 (1988) 237(E)

[9] A.S.Carnoy et.al., J. Phys. G 18 (1992) 823

[10] J.Ashman et.al. Phys. Lett. B206 (1988) 364; J.Ashman et.al. Nucl. Phys. B328 (1989) 1

[11] J.Ellis and R.L.Jaffe, Phys. Rev. D9 (1974) 1444r

[12] J.Kodaira et.al. Phys. Rev. D20 (1979) 627; Nucl. Phys. B159 (1979) 99; J.Kodaira Nucl. Phys. B165 (1980) 129

[13] J.T.Londergan and S.Kumano in "Spin and Isospin in Nuclear Interactions", Ed. by S.W.Wissink, C.D.Goodman, G.E.Walker, Plenum Press, N.Y., 1991, 1

[14] M.Shmatikov, Yad. Fiz. (Sov. Journ. Nucl. Phys.) 54, 6(12) (1991) 1677

[15] M. Bourquin et.al Z.Phys C21 (1983) 17

[16] W. Tanenbaum et.al Phys. Rev. D12 (1975) 1871

[17] P.L.Anthony et.al. Phys. Rev. Lett., 71, (1993) 959

[18] D.B.Kaplan, A.Manohar, Nucl. Phys. B310 (1988) 527

[19] C.A.Dominguez, Paul Langacker Phys. Rev. D24 (1981) 1905

[20] E.Reya Rev.Mod.Phys. 46 (1974) 545

[21] J.Gasser, H.Leutwyler and M.Sainio, Phys. Lett B253 (1991) 252

[22] J.Rapaport, In Proc. IUCF Workshop "Interaction between Medium Energy Nucleons in Nuclei", Preprint, Ohio University (1982)

[23] C.Gaarde et.al., Nucl.Phys. A369 (1981) 258

[24] W.Weise, In. Progress in Particle and Nuclear Physics, v. 11 (1983) 123

[25] S.L.Adler Phys. Rev. 140 (1965) B736;

[26] W.I.Weisberger, Phys. Rev. 143 (1966) 1302

[27] Yu.S Lutostansky, N.B.Shul'gina, Phys. Rev. Lett. 67 (1991) 430 